\title{
Los cambios recientes en el entorno estratégico de la Unión Europea con especial atención al papel de Rusia*
}

\author{
The recent changes in the strategic environment of the European Union: \\ A case study of Russia \\ Antonio Blanc Altemir \\ Catedrático de Derecho Internacional Público y Relaciones Internacionales. \\ Universidad de Lleida \\ Titular de la Cátedra Jean Monnet: «Law and External Relations of the European Union», \\ y Director del Centro de Excelencia Jean Monnet. Comisión Europea-UdL
}

\begin{abstract}
Sumario: I. Consideraciones preliminares: La UE ante las amenazas y los desafíos en su entorno estratégico.-II. El conflicto de Ucrania y su capacidad para amenazar el entorno estratégico de la UE. 1. La respuesta de Rusia a la destitución de Yanukovich: la anexión de Crimea y la presión en el Este de Ucrania. A. La anexión de la península de Crimea. B. El conflicto en el Este-Sur de Ucrania. 2. De Minsk I (5 de septiembre de 2014) a Minsk II (12 de febrero de 2015)._III. Impacto del conflicto de Ucrania en las relaciones de Rusia con la UE y con la OTAN. 1. Impacto en las relaciones de la Federación Rusa con la Unión Europea. 2. Impacto en las relaciones de la Federación Rusa con la OTAN.-IV. Conclusiones
\end{abstract}

\begin{abstract}
Resumen: Las nuevas amenazas en el entorno estratégico de la Unión se centran en dos frentes diferenciados. En primer lugar en su flanco Sur, determinado por un Mediterráneo siempre inestable en el que persisten conflictos no superados y en el que confluyen otros escenarios crecientemente inestables como el Sahel y Oriente Medio. En segundo lugar, en su flanco Este, en el que la anexión de Crimea por parte de Rusia y el apoyo de ésta a los separatistas del Donbass, determina un escenario amenazador para la estabilidad de la UE, que le ha obligado a redefinir sus relaciones con su vecino del Este, adoptando una posición que intenta combinar una actitud de firmeza, con un intento de no romper todos los canales de comunicación con Rusia.
\end{abstract}

Palabras clave: Ucrania; Donbass; Crimea; Rusia; UE.

Abstract: The new threats that the European Union is facing are located mainly in its two strategic environments. Firstly, the southern edge characterised by an unstable Mediterranean where there are non-resolved conflicts as well as other scenarios of increasing instability such as the Sahel and the Middle East. Sec-

* Recibido el 31 de marzo de 2015, aceptado el 30 de mayo de 2015. 
ondly, the eastern edge where Russia has annexed Crimea and supports the separatists of Donbass pointing to a risky scenario for the stability of the EU. Due to that fact, the Union has redefined its relationships with the eastern neighbours adopting a position that combines an assertive approach with an attempt of not breaking the channels of communication with Russia

Keywords: Ukraine; Donbass; Crimea; Russia; EU.

\section{Consideraciones preliminares: La UE ante las amenazas y los desafíos en su entorno estratégico}

A pesar de los éxitos diplomáticos, limitados pero indiscutibles, de la última fase de la «etapa Ashton», como son la firma en 2013 del acuerdo entre Serbia y Kosovo para la normalización de las relaciones recíprocas, y el acuerdo provisional entre Irán y el Grupo P5 + 1 (los cinco miembros permanentes del Consejo de Seguridad, más Alemania), la llegada de la nueva Comisión Europea ha coincidido sin lugar a dudas con una tendencia «a la baja» en la capacidad europea para incidir en el escenario internacional. El pecado original de la apuesta inicial por un bajo perfil, unido a las conveniencias tácticas y al protagonismo de los grandes Ministerios de Exteriores de Alemania, Francia y Reino Unido, han marcado en buena parte la política exterior europea de estos últimos años, sin que pueda ignorarse, y mucho menos menospreciarse, la puesta en marcha del Servicio Europeo de Acción Exterior (SEAE).

El acceso de la antigua Ministra de Exteriores italiana Federica Mogherini al cargo de Alta Representante de la Unión para Asuntos Exteriores y Política de Seguridad coincide con la aparición o intensificación de nuevas amenazas en el entorno estratégico de la Unión que marcarán los próximos años. Se trata del flanco Sur, determinado por un Mediterráneo siempre inestable en el que persisten conflictos no superados desde la aparición de la primavera árabe, y en el que confluyen otros escenarios crecientemente inestables como el Sahel y Oriente Medio; y del flanco Este, en el que la anexión de Crimea por parte de Rusia y el apoyo de ésta a los separatistas del Donbass, determina un escenario verdaderamente amenazador para la estabilidad de la Unión Europea.

Con independencia de que ambos desafíos son muy diferentes, el hecho de que se manifiesten simultáneamente: la amenaza terrorista yihadista que se cristaliza en particular en la aparición del Estado Islámico y la irrupción de una Rusia revisionista, quizás representan la expresión de un síntoma, que sería la debilidad de Occidente en general, y de la UE en particular, cuyas vacilaciones y divisiones respecto a cómo enfrentarlos, habrían sido aprovechados por ambos. 
Por lo que respecta al primero de los desafíos en el entorno estratégico de la Unión, el relativo a su flanco Sur, conviene recordar que la amenaza yihadista se está extendiendo muy rápidamente en el Magreb y en el Sahel, como lo demuestra el incremento de ataques terroristas en ambas zonas que, por otra parte, presentan una indiscutible continuidad geográfica.

El incremento de la capacidad de captación de los grupos terroristas en la región, ha tenido igualmente un impacto considerable en la capacidad operativa de los grupos yihadistas, lo que unido a otros factores como el crimen organizado y los conflictos armados constituyen una auténtica amenaza para la estabilidad del Mediterráneo y de la propia UE ${ }^{1}$. La irrupción del Estado Islámico (EI) como una amenaza mucho mayor que la que haya representado Al Qaeda, al controlar de forma efectiva una parte no despreciable de territorio, y al contar con una amplia financiación, armas sofisticadas y un mando militar competente, determinó la formación de una coalición internacional auspiciada por Estados Unidos con el objetivo de frenar el desafío del EI.

El método utilizado por dicha coalición, basado en los bombardeos aéreos sistemáticos, logró dar sus frutos en un principio al frenar el fulgurante avance del califato recuperando algunos pozos petrolíferos iraquíes como Biyi que había caído en manos del EI y dando apoyo logístico y militar al Ejército regular iraquí y a los «peshmergas» en el progresivo asedio de Mosul, tercera ciudad iraquí que cayó en manos del EI en junio de 2014 y cuya recuperación constituye un objetivo estratégico de la coalición. Sin embargo, el hecho de que varias organizaciones islámicas radicales que operan en Libia y Egipto, hayan jurado fidelidad al Estado Islámico, constituye un elemento preocupante pues supone una amenaza cierta para el entorno estratégico de la Unión, por varias razones: en primer lugar porque al contrario de $\mathrm{Al}$ Qaeda, que funciona como una especie de franquicia que acoge en su seno a cualquier individuo o grupo con capacidad de llevar a cabo ataques que coincidan con sus intereses, el EI dispone en las zonas sometidas a su control, de una auténtica estructura administrativa capaz de mantener su propia burocracia, pagar salarios, explotar los pozos petrolíferos y vender sus derivados, además de dotarse de su propio instrumento financiero, basado en el patrón oro. En segundo lugar, porque extendiendo sus tentáculos hasta Egipto y Libia, la influencia del EI llega hasta orillas del Mediterráneo, aprovechando las dificultades

${ }^{1}$ Sobre las amenazas provenientes del flanco Sur, vid. DÍEZ ALCAIDE, J., «La seguridad del Sur: una decisión apremiante para la cumbre de la OTAN», Instituto Español de Estudios Estratégicos, Documento Análisis, n. ${ }^{\circ}$ 39/2014, de 30 de julio de 2014, pp. 5 y ss. 
del Gobierno de Egipto para controlar efectivamente la península del Sinaí y el caos existente en Libia $^{2}$.

Además de este escenario de conflictividad que afecta directamente a nuestro entorno de seguridad inmediata, la grave crisis de Ucrania, en la que vamos a centrar nuestro análisis, amenaza con revisar el orden internacional y concretamente europeo, derivado de la desintegración de la Unión Soviética.

\section{El conflicto de Ucrania y su capacidad para amenazar el entorno estratégico de la UE}

\section{La respuesta de Rusia a la destitución de Yanukovich: la anexión de Crimea y la presión en el Este de Ucrania}

Desde el inicio de las protestas en Kiev a finales de noviembre de 2013 hasta el anuncio de la huida de Yanukovich el 22 de febrero de $2014^{3}$, se produjeron una serie de acontecimientos que se precipitarían a partir de esta fecha provocando una reacción múltiple: por un lado los manifestantes del Maidán tomaron la sede de la presidencia del Gobierno; y por otro, la Rada Suprema adoptó una serie de medidas de gran calado, como la liberación de la opositora Yulia Timoshenko y la destitución del presidente Yanukóvich por haber dejado de cumplir «sus competencias constitucionales, lo que amenaza la gobernabilidad del Estado y la integridad territorial y la soberanía de Ucrania, y la transgresión masiva de los derechos y libertades de los ciudadanos».

La destitución de Yanukóvich, aunque constitucionalmente muy discutible, propició la emergencia de un polo de oposición en torno a la ciudad de Jarkov, donde se refugió en un primer momento Yanukóvich, aglutinando las regiones rusoparlantes del este y sur de Ucrania que interpretaron la destitución como un golpe de Estado propiciado por los extremistas del

2 Aprovechando la ausencia de autoridad estatal y la porosidad de las fronteras, grupos radicales islámicos, que han jurado lealtad al EI, tomaron en la primavera de 2014 la ciudad libia de Derna, situada en el Este del país, y más recientemente la ciudad de Naufaliya, situada al Norte a 120 Kilómetros de Sirte.

3 Para un análisis detallado de los acontecimientos acaecidos hasta la destitución de Yanukovich, que no podemos abordar por razones de espacio, vid. RUIZ GONZÁLEZ, F., «Ucrania: revolución y guerra civil. Una visión alternativa de la crisis». Instituto Español de Estudios Estratégicos. Documento Marco, $n .^{\circ}$ 19/2014, 13 de noviembre de 2014, y FARAMIÑAN GILBERT,J.M., «Ucrania, sobre la línea roja». Real Instituto ElcaNo. Documento de Trabajo, n. ${ }^{\circ}$ 17/2014, 30 de diciembre de 2014; BERMEJO GARCÍA, R., .La vuelta de Crimea a la madre-patria. Tirant lo Blanch, Valencia, 2015, en especial pp. 32-55. 
Maidán, coincidiendo en su apreciación con Rusia, a donde se dirigiría el destituido expresidente. Paradójicamente, la UE que había auspiciado el acuerdo del día anterior, lo ignoró completamente al reconocer rápidamente a las nuevas autoridades de Kiev, que, sintiéndose apoyadas por Occidente cometieron dos errores de gran calado para el devenir del país: en primer lugar marginaron totalmente al Partido de las Regiones en la configuración de los nuevos puestos clave del nuevo Gobierno y, en segundo lugar, derogaron la ley, adoptada en 2012, por la que se declaraba el ruso como idioma oficial en aquellas regiones que superara el $10 \%$ de utilización entre su población ${ }^{4}$.

Sin embargo, las protestas surgidas en Donetsk, en el oeste del país y en Crimea - donde empezaban a surgir las primeras tensiones separatistas - contra las nuevas autoridades de Kiev, hacían presagiar que las tensiones entre el Este, de influencia rusa, y el Oeste proeuropeo, serían cada vez más visibles, y que Rusia no adoptaría una posición neutral, como lo ponía de manifiesto la exhibición de su fuerza militar ante Ucrania al poner en alerta las circunscripciones militares del centro y oeste de Rusia. De esta forma, se abría un nuevo capítulo del duelo geopolítico entre Rusia y Occidente que pondría a la península de Crimea en el centro de las turbulencias inmediatas para continuar casi de forma simultánea con el Este de Ucrania.

En efecto, tras la constitución de un Gobierno de unidad nacional en Kiev, liderado por Arseni Yatseniuk, Rusia manifestó su rechazo frontal al mismo calificándolo de sectario e ilegítimo y acusando a Occidente de haber apoyado a las fuerzas radicales que, mediante un «golpe de Estado», finalmente se habían hecho con el poder. Como respuesta a esta nueva situación, Rusia adoptó una estrategia de presión al nuevo régimen de Kiev, temerosa tanto de que su proyecto de Unión Euroasiática quedara vacío sin la participación de Ucrania, como de que la revolución democrática triunfara definitivamente en el país, con la posibilidad de contagio a sus propias fronteras.

La estrategia de Putin, cuidadosamente planificada para aumentar la presión sobre Ucrania, tendría dos escenarios complementarios, en los que la influencia rusa estaba fuera de toda duda: la península de Crimea y el Este-Sur de Ucrania.

\section{A. La anexión de la península de Crimea}

A la alerta fronteriza de las tropas rusas, siguió la ocupación del Parlamento de Crimea, de la sede del Consejo de Ministros, de la televisión y de

${ }^{4}$ Decisión que finalmente sería vetada por el presidente en funciones Alexander Turchinov. 
los nudos de comunicaciones por rusófilos armados, pero sin identificación oficial $^{5}$. El control de los edificios públicos fue acompañado de una neutralización de la capacidad operativa de las mermadas fuerzas ucranianas, a través del control de las dos principales carreteras que unen la península con el resto de Ucrania, de los aeropuertos internacionales de Simferópol y Sebastopol así como de las bases aéreas, al mismo tiempo que se rodeaban las principales instalaciones militares.

La presión continuaría con la rápida aprobación por parte de la Duma rusa de la petición del presidente Putin para desplegar tropas en Ucrania, lo que incluía no sólo la península de Crimea sino también el Este y Sur del país, mayoritariamente rusohablante y a cuya defensa apelaba Putin, en caso de que el nuevo Gobierno de Kiev adoptara medidas que no tuvieran en cuenta los intereses de los millones de prorrusos que habitan en dichas zonas. La reacción del Gobierno de Kiev ante la expansión militar rusa en la península y la impotencia ante las deserciones de miembros destacados del Ejército ucraniano en Crimea, fue poner al Ejército en estado de alerta de combate y decretar la movilización de los reservistas.

Mientras tanto, el Parlamento de Crimea solicitó, el 6 de marzo, a la Duma Estatal (Cámara Baja del Parlamento ruso) y al Presidente Putin, la incorporación de la península a la Federación Rusa y decidió adelantar el referéndum sobre el futuro de Crimea, inicialmente convocado para el 25 de mayo, al 16 de marzo. La respuesta, previsible por otra parte, de las dos cámaras del Parlamento ruso a dicha petición fue positiva pues en una declaración conjunta de sus presidentes constataron que Rusia aceptaría a Crimea como una república más de la Federación, si la población de la península se manifestaba positivamente en el referéndum del 16 marzo.

Las reacciones provocadas por la convocatoria del referéndum se polarizaron en torno a dos diferentes: la de la UE, Estados Unidos y países aliados que manifestaron su rechazo frontal a la consulta por considerarla ilegal de acuerdo con la legislación interna de Ucrania y el Derecho internacional; y la de Rusia que la consideraba absolutamente legal y ajustada al ordenamiento jurídico internacional. En efecto, tanto las instituciones de la UE, Comisión, Parlamento y Consejo, como la OTAN, la OSCE, y los respectivos países miembros, advirtieron a Rusia que no reconocerían el resultado del referéndum por considerarlo ilegal, según la Cons-

${ }^{5}$ Según todos los indicios, se trataba tanto de tropas de intervención especial (Berkut) de Sebastopol, como de una parte de los restos de la Berkut ucraniana depuestas de sus funciones por las nuevas autoridades de Kiev. Posteriormente se unieron grupos de cosacos, tanto locales como llegados de otras partes de Rusia, además de grupos de autodefensa crimeos. 
titución de Ucrania que no acepta las consultas locales o regionales ${ }^{6}$, así como una amenaza para la seguridad y estabilidad internacional. En cambio Rusia alegaba el precedente de la escisión de Kosovo, reconocido por un amplio número de Estados de la UE y de la comunidad internacional, aunque no por la propia Rusia, y la legalidad de la consulta que basaba en la Carta de las Naciones Unidas, y en particular en su artículo 1, que reconoce el principio de igualdad de derechos y de libre determinación de los pueblos.

El paralelismo que Rusia alegaba entre los casos de Kosovo y Crimea, que llevaban al Presidente Putin y a su Ministro de Asuntos Exteriores Serguéi Lavrov a afirmar que «si Kosovo era un caso especial, Crimea también es un caso especial» ${ }^{7}$, presenta sin embargo algunas reservas que conviene señalar. Es cierto que en ambos casos nos encontramos con partes de un territorio estatal del que decidieron separarse unilateralmente, y que también en ambos supuestos se trata de una población asentada en dicho territorio con una mayoría étnica diferente a la dominante en el Estado matriz. Además, en ambos casos se ha dado una intervención militar externa sin autorización previa del Consejo de Seguridad de las Naciones Unidas, y ambos se han producido en clara violación del Derecho interno del Estado al que pertenecían (Serbia y Ucrania).

Existen, no obstante, diferencias remarcables como es el hecho relevante de que en Kosovo, Serbia perpetró violaciones masivas y sistemáticas de los derechos humanos que fueron calificadas de limpieza étnica, hecho que no se produjo en Crimea por parte de Ucrania. Esta circunstancia justificaría para algunos la excepcionalidad de Kosovo, o al menos le concedería una legitimidad moral. Es cierto que podemos aceptar esa «legitimidad moral» que el caso de Crimea no presenta, pero la legalidad internacional parece estar fuera de ambas situaciones. En Kosovo por la existencia de la Resolución 1244 del Consejo de Seguridad que garantizaba la integridad territorial de Serbia, cuya agresión a la población kosovar se produjo nueve años antes de la declaración de independencia unilateral de Kosovo, cuyo territorio estaba bajo el mandato de Naciones Unidas en ese momento. En

${ }^{6}$ El propio Tribunal Constitucional de Ucrania declaró que el referéndum era inconstitucional.

7 Tanto Putin en sus conversaciones telefónicas con Obama u otros dirigentes occidentales, en particular de la UE, como el Ministro de Asuntos Exteriores Lavrov utilizaron frecuentemente este paralelismo. En el encuentro que los jefes de las diplomacias norteamericana y rusa, Kerry y Lavrov, mantuvieron en Londres para acercar posiciones sobre la crisis de Ucrania y la situación en Crimea, Lavrov volvió a utilizar el precedente de Kosovo como justificación de la actuación rusa en Crimea, añadiendo que «Crimea es muchísimo más importante que las Malvinas para Reino Unido o las Comoras para Francia». Cfr. El País,15 de marzo de 2014, p. 3 . 
Crimea por las circunstancias que rodearon la intervención rusa, la convocatoria y realización del referéndum y la posterior anexión.

Los días que precedieron a la realización del referéndum fueron igualmente de gran tensión en el ámbito defensivo. En efecto, Rusia inició nuevas maniobras militares junto a la frontera ucraniana en las que participaron unos 10.000 soldados, y envió a su aliada Bielorrusia seis cazas y tres aviones de transporte para la vigilancia del espacio aéreo. Ante esta escalada que suponía un desafío en toda regla si lo asociamos con la situación en Crimea y con el apoyo a los separatistas del Este y Sur de Ucrania, la OTAN acordó desplegar dos aviones AWACS de vigilancia de fronteras para vuelos de reconocimiento sobre Rumania y Polonia, dos países con fronteras con Ucrania. Además Estados Unidos envió 12 cazas F-16 a Polonia y otros seis F-15 a Lituania, e inició maniobras navales conjuntas con las armadas de Rumania y Bulgaria en el Mar Negro. El «frente defensivo» se hacía por lo tanto cada vez más patente, como lo demostraba el hecho de la mayor presencia pública de la OTAN en el conflicto, así como la suspensión de algunas actividades militares conjuntas con Rusia, aunque manteniendo la línea de diálogo.

Sin embargo, nada pudo evitar la celebración del referéndum el 16 de marzo, ni tampoco el proyecto de resolución presentado ante el Consejo de Seguridad el día anterior por Estados Unidos - y obviamente vetado por Rusia - en el que se remarcaba la integridad territorial de Ucrania y se condenaba expresamente el referéndum en Crimea por considerarlo ilegítimo.

El referéndum lo ganó ampliamente, según todas las previsiones, la opción de la incorporación a Rusia $(96,77 \%)$, obteniendo la opción de continuar en Ucrania pero en el marco de la Constitución de Crimea de 1992, el $2,51 \%{ }^{8}$. Curiosamente ninguna de las dos preguntas incorporadas a la consulta incluía la opción de mantener el statu quo. La participación alcanzó el $83,1 \%$ del censo. Llama la atención en este sentido, que aunque la mayoría de la población es de etnia rusa (58\%), siendo los ucranianos el $24 \%$ y los tártaros el 13\%, el resultado fuera tan mayoritario a favor de la incorporación a Rusia, lo que demostraría que una parte importante de la población de etnia ucraniana, pero de lengua rusa, votó igualmente por dicha opción. En cambio, la comunidad tártara (cerca de 300.000 personas), se había manifestado a favor del boicot, a través del Medzhlis, que constituye el órgano

8 A la primera pregunta: «¿Está usted a favor de la reunificación de Crimea con Rusia en los derechos de la Federación Rusa?» contestaron el 96,77\% a favor, y a la segunda pregunta: «¿Está usted a favor de que se restablezca la Constitución de la República de Crimea de 1992 y el estatus de Crimea como parte de Ucrania previsto en ella?», contestaron a favor tan sólo el 2,51\%. Cfr. Fondation Robert Schuman, La Carta, n. ${ }^{\circ}$ 617, 18 de marzo de 2014. 
ejecutivo del Qurultai, la Asamblea tártara de Crimea, que es un órgano paralelo a las instituciones oficiales de Crimea.

Realizado el recuento, empezó el proceso de incorporación formal de Crimea a Rusia con la declaración de independencia realizada por el Parlamento de Crimea ${ }^{9}$, que venía a ratificar la inicial declaración unilateral de independencia efectuada el 11 de marzo, es decir cinco días antes del referéndum, como paso previo y coordinado con Rusia para evitar que pudiera ser considerado como una simple anexión. La declaración de independencia efectuada el día posterior al referéndum, es decir el 17 de marzo, sustituía la República Autónoma de Crimea (integrada en Ucrania), por la República (independiente) de Crimea, y tras hacer un llamamiento a las Naciones Unidas y a todos los países del mundo para solicitar el reconocimiento como Estado independiente, solicitaba la inclusión a Rusia como República de la Federación. La declaración pretendía allanar el camino para su integración en Rusia a través de un acuerdo internacional bilateral, adoptado y firmado por dos sujetos con subjetividad jurídica internacional plena, según esta perspectiva, como eran Rusia y Crimea ${ }^{10}$.

La formalización del proceso de fusión requería el reconocimiento de la independencia de Crimea por parte de Rusia, la firma del tratado bilateral, que debería ser sometido al Tribunal Constitucional de la Federación Rusa, y posterior votación de las dos cámaras del Parlamento ruso (Duma y Senado) y a la firma del Presidente ruso. Trámites todos ellos que se llevaron a cabo con una rapidez inusitada, por lo que el 18 de marzo, y por lo tanto tan sólo dos días después del referéndum, la anexión-fusión era efectiva con la firma del tratado bilateral entre el presidente Putin, el primer ministro de la flamante república de Crimea, el presidente del Parlamento de Crimea y el alcalde de Sebastopol, a la espera del trámite de la aprobación del Parlamento y del Tribunal Constitucional ruso.

A la vista del procedimiento «exprés» utilizado en este caso, cabe preguntarse si podemos considerarlo o no acorde con el Derecho internacional. Es cierto que el Presidente ruso utilizó como argumento jurídico, no sólo el precedente de Kosovo que ya hemos comentado, sino también y de forma particular el derecho de libre determinación de los pueblos reconocido expresamente por la Carta de las Naciones Unidas, pero hay que consi-

${ }^{9}$ La declaración fue votada por 85 diputados de un total de 100 escaños que tenía el Parlamento de Crimea.

${ }^{10}$ Se establecía igualmente que la grivnia ucraniana sería sustituida por el rublo ruso, de forma paulatina (hasta enero de 2016), en un proceso que controlará el nuevo Banco de Crimea. La hora de Kiev sería igualmente sustituida por la hora de Moscú a partir del 30 de marzo, y la ciudad de Sebastopol - base de la flota rusa - pasaría a tener un estatus especial, como Moscú y San Petersburgo. 
derar que dicho principio se reconoce explícitamente a los pueblos sometidos a dominación colonial, o, excepcionalmente, a aquéllos que son objeto de violaciones masivas y sistemáticas de los derechos humanos, o que sean tratados de forma abusiva, discriminatoria y sin el mínimo respeto a la representación democrática por parte del Gobierno del Estado del cual pretenden independizarse ${ }^{11}$, lo que en ningún caso sería aplicable al caso de Crimea. Además, conviene señalar que en el Derecho internacional existe igualmente el principio de la integridad territorial que en este caso prevalecería sobre el de libre determinación y constituiría un claro límite a la aplicación de éste ${ }^{12}$. Por otra parte, tampoco son totalmente aceptables desde la perspectiva jurídica internacional, los argumentos esgrimidos por Rusia sobre que el pueblo de Crimea se pronunció libremente en un referéndum pretendidamente imparcial y transparente, y que el TIJ aceptó como legal la secesión de Kosovo en su controvertida Opinión consultiva de 22 de julio de $2010^{13}$.

\section{B. El conflicto en el Este-Sur de Ucrania}

Cuando todavía no había desaparecido el contundente impacto producido por la anexión de Crimea por parte de Rusia, miles de activistas prorrusos ocuparon, el 6 de abril de 2014, varias sedes gubernamentales en las ciudades de Donestk, Jarkov y Lugansk, sin que las fuerzas de seguridad pudieran impedirlo. La efectividad y rapidez de la ocupación por parte de

${ }^{11}$ Una parte de la doctrina distingue en este sentido entre las dimensiones externa e interna del principio, vinculando esta última al llamado principio democrático. Vid. a tal efecto PONS RÄFOLS, X. «Legalidad internacional y derecho a decidir», Revista Electrónica de Estudios Internacionales, 2014, pp. 21 y s.s.; BERMEJO GARCÍA, R., La vuelta de Crimea...», op. cit., nota 3 , pp. 84 y s.s.

${ }_{12}$ Coincidimos plenamente en esta apreciación con el profesor Pons Ràfols. Cfr. PONS RÀFOLS, X., «Maniobras ilegales contra Ucrania», El País, 19 de marzo de 2014, p. 29; PONS RÀFOLS, X., «Legalidad internacional y derecho a decidir», op. cit., nota 11, pp. 35 y s.s. En este mismo sentido, vid. ACOSTA SÁNCHEZ, M.A., «La secesión en el Derecho internacional: el caso de Crimea». Instituto Español de Estudios Estratégicos, Documento Opinión 142/2014, 11 de diciembre de 2014, pp. 4 y s.s.

${ }^{13}$ Sobre esta cuestión vid.: GUTIÉRREZ ESPADA, C. y BERMEJO GARCÍA, R., De la Opinión consultiva de la CIJ, de 22 de Julio de 2010, sobre Kosovo, Real Instituto Elcano, Doc. de Trabajo n. ${ }^{\circ}$ 35/2010, de 10/11/2010. pp. 1-13; BERMEJO GARCÍA, R. y GUTIÉRREZ ESPADA, C., «La declaración unilateral de independencia de Kosovo a la luz de la Opinión consultiva de la Corte Internacional de Justicia, de 22 de Julio de 2010, y de las declaraciones, opiniones individuales y disidentes a la misma», Anuario Español de Derecho Internacional, t. 26, 2010, pp. 7-59; Revista Española de Derecho Internacional, n. ${ }^{\circ} 12011$, número monográfico dedicado al análisis de dicha Opinión consultiva, con estudios de los profesores C. Escobar Hernández; C. Jiménez Piernas; P. Andrés Sáenz de Santa María, J. Cardona Llorens; A. Mangas Martín y C. Espósito Massicci. 
los activistas, así como el desafío lanzado hacia el gobierno central de Kiev, declarando la independencia de la región y convocando un referéndum de adhesión a Rusia para el 11 de mayo, provocaron una honda preocupación no sólo en el resto del país, todavía traumatizado por la inestabilidad provocada por el Maidán y la posterior pérdida de Crimea, sino también en la UE y en el resto de la comunidad internacional.

En un principio todo parecía indicar que se trataba de un nuevo Maidán, aunque de signo contrario al protagonizado en Kiev unos meses antes, pues los activistas prorrusos se hicieron fuertes primero en Donetsk (donde proclamaron la República Popular de Donetsk), y después en otras ciudades como Lugansk y Jarkov, ocupando edificios públicos, tomando las armas allí depositadas y proclamando su independencia de Kiev al mismo tiempo que se solicitaba ayuda a Rusia para resistir la más que previsible respuesta del Gobierno central ${ }^{14}$. Aunque las autoridades lograron retomar el control de los edificios ocupados, entre ellos la sede de la Administración regional, en Jarkov, segunda ciudad del país, sin embargo la situación se enquistó en Donetsk y Lugansk, donde los activistas llegaron a minar el edifico del Servicio de Seguridad tomando a más de sesenta rehenes.

La reacción de Kiev ante estos acontecimientos fue de un cierto desconcierto en un principio, aunque la Rada Suprema elevó rápidamente las penas por los delitos de traición al Estado, espionaje y atentado contra la integridad territorial del país, al mismo tiempo que el Ministerio de Asuntos Exteriores ucraniano acusaba a Rusia de continuar con la agresión perpetrada en una primera fase en Crimea y de alentar a los movimientos separatistas en el Este del país. Sin embargo la respuesta de Rusia no fue muy tranquilizante pues ignoró la petición de retirar sus tropas situadas cerca de la frontera con Ucrania ${ }^{15}$ exigiendo al mismo tiempo la federalización del país, la cooficialidad del idioma ruso y un estatus de neutralidad que impidiera el ingreso de Ucrania en la OTAN ${ }^{16}$.

Tras la visita del primer ministro Yatseniuk a Donetsk en la que prometió más autonomía a la región pero reafirmando la integridad territorial del Estado ucraniano como principal objetivo de su gobierno, y ante la determinación del Consejo de la autoproclamada República Popular de Donetsk,

14 Entre la docena de grupos de activistas se daba un cierta heterogeneidad, pues convergían desde los más radicales que exigían la independencia y la adhesión a Rusia, hasta otros más moderados que exigía un modelo federal para Ucrania así como el uso oficial de la lengua rusa en las regiones del Este y del Sur del país.

15 Negándose al efecto a participar, en el marco de la OSCE, en las consultas sobre la actividad militar en sus fronteras.

${ }^{16} \mathrm{El}$ anterior presidente Yanukovich, promulgó una ley en 2010 en la que excluía la posibilidad de que Ucrania ingresara en la OTAN y reafirmaba su neutralidad. 
erigido como órgano colectivo coordinador de las protestas en dicha región, de no dar marcha atrás en la ocupación de los edificios oficiales, el presidente en funciones, Alexandr Turchínov, firmó el decreto en Kiev que ponía en marcha la operación antiterrorista, que con la participación del Ejército, intentaría desalojar a los activistas y controlar una situación que cada vez se mostraba más compleja. Mientras tanto, los rebeldes se negaban a reconocer la autoridad del presidente en funciones Turchínov y del primer ministro Yatseniuk, y rechazaban la convocatoria de elecciones presidenciales en el país para el 25 de mayo, al mismo tiempo que insistían en la celebración de un referéndum de autodeterminación para el 11 de mayo, que en ningún caso reconocía la legislación ucraniana.

Las conversaciones celebradas en Ginebra el 17 de abril con la participación de Estados Unidos, Rusia, la UE y Ucrania, dieron como resultado un principio de acuerdo ${ }^{17}$ sobre la base de frenar la violencia y la inestabilidad, para lo cual todos los grupos armados ilegales deberían ser desarmados, los edificios oficiales desalojados, garantizándose una amnistía para los rebeldes que hubieran ocupado edificios, plazas o calles, salvo que hubieran cometido delitos muy graves. Como contrapartida, el Gobierno central se comprometía a abrir un diálogo nacional conducente a otorgar mayores competencias a las regiones y a aceptar un proceso de descentralización que reconociera la diversidad del país. Ambas partes se comprometieron igualmente a dar un gran protagonismo a los observadores internacionales, en particular a la OSCE que ya se hallaba sobre el terreno $^{18}$, con el fin de asistir a las partes enfrentadas en la aplicación y verificación del acuerdo.

Sin embargo, la multiplicación de incidentes armados en Slaviansk y otras ciudades como Kramatorsk, en las que se produjeron víctimas mortales durante los días siguientes a su adopción, pusieron de manifiesto la fragilidad de los acuerdos de Ginebra en un escenario en el que Rusia, que seguía negando su implicación en la revuelta, denunciaba al gobierno de Kiev de violar tales acuerdos al no concederse la amnistía pactada a los activistas prorrusos, y por no aplicarlos a las milicias del Maidán en la propia capital.

Con la intensificación de los incidentes cada vez más violentos, como la captura de ocho inspectores militares de la OSCE por parte de las milicias prorrusas, acusados por éstos de ser «espías de la OTAN»o el incendio intencionado en un edificio oficial de Odessa durante una manifestación

17 Cfr. European Union. External Action, Geneva Statement on Ukraine, Geneva, 17 April 2014, Doc. 140417/01.

${ }^{18}$ En el marco de la Misión Especial de Monitorización que debería desplegarse a continuación de tales acuerdos. 
que produjo treinta y seis muertos ${ }^{19}$, los acuerdos de Ginebra pronto serían considerados como «papel mojado» por las partes implicadas cuyas posiciones fueron progresivamente radicalizándose con enfrentamientos armados directos en un escenario en el que Rusia acusaba al gobierno provisional de Kiev de usar armas pesadas y carros de combate contra civiles y a Occidente de apoyar tales medidas. Al mismo tiempo, Rusia ordenaba maniobras militares en la frontera con Ucrania, lo que disparó la tensión provocando un deterioro todavía más intenso en las relaciones bilaterales rusoucranianas, en un contexto de violencia progresiva en el que el presidente ucraniano admitió que las provincias de Donetsk y Lugansk estaban fuera del control de las autoridades de $\mathrm{Kiev}^{20}$.

La liberación de los inspectores militares de la OSCE, tras la presión occidental ejercida desde su captura, así como la petición del presidente ruso Vladimir Putin a los separatistas de que aplazaran el referéndum convocado para el 11 de mayo, al mismo tiempo que aseguraba haber retirado las tropas desplegadas en la zona fronteriza, avalaba las elecciones del 25 de mayo en Ucrania y solicitaba el cese de las operaciones de castigo del Gobierno de Kiev en el sudeste ucraniano, pareció facilitar una cierta distensión en el conflicto. Tras el desconcierto inicial no sólo en las filas de los activistas prorrusos, que ya tenían organizado el referéndum con las papeletas incluso impresas, sino también en las cancillerías occidentales, los líderes de la autoproclamada República de Donetsk decidieron mantener la convocatoria, desoyendo la petición del presidente ruso.

A pesar de que la legislación interna de Ucrania no contempla tal tipo de consultas y de que tanto la UE como Estados Unidos lo consideraron ilegal por carecer de garantías, de transparencia y de los mínimos mecanismos

19 La tragedia se produjo en el marco de los enfrentamientos entre prorrusos y partidarios de la unidad de Ucrania, en el mayor estallido de violencia en la ciudad ucraniana de Odessa, de mayoría rusa, en el mar Negro. El incendio intencionado, provocado por el lanzamiento de artefactos explosivos a la Casa de los Sindicatos en el que se refugiaron los prorrusos tras «reventar» una marcha en favor de la unidad de Ucrania, causó treinta y seis muertos, a los que se unieron otros seis en los enfrentamientos ocurridos en las calles de la ciudad.

${ }^{20}$ En los alrededores de Slaviansk, núcleo industrial de unos 130.000 habitantes y avanzadilla de la revuelta contra Kiev, quedó patente ya iniciado el mes de mayo que la operación «antiterrorista» (en la que participaba el Ejército apoyado por la Guardia Nacional y dos unidades de las fuerzas especiales antiterroristas Alfa), lanzada por el Gobierno de Kiev para recuperar el control sobre el este rebelde, tenía serios problemas para lograr tal objetivo. El cerco que el Ejército ucraniano sometió a Slaviansk se saldó con numerosas bajas en las filas gubernamentales mientras que un helicóptero militar, un Mi-24, caía abatido por la artillería rebelde, siendo el tercer aparato derribado desde que comenzaron las operaciones militares, lo que demostraba la progresiva organización de los activistas prorrusos así como su acceso a armas cada vez más sofisticadas. 
de control, el referéndum se realizó en la fecha señalada, 11 de mayo, y su resultado, tal como estaba previsto, fue mayoritariamente a favor de la secesión de Ucrania, tanto en Donetsk como en Lugansk ${ }^{21}$, en una jornada en la que los combates continuaron y en la que no hubo observadores internacionales. En un proceso que recordaba la anexión de Crimea escasamente dos meses antes, los líderes rebeldes propusieron la anexión a Rusia y su negativa a participar en los comicios presidenciales a celebrar el 25 de mayo en Ucrania.

Celebradas en la fecha prevista, las elecciones presidenciales fueron ampliamente ganadas en la primera vuelta con un 55,9\% de votos, por el candidato independiente Petro Porochenko, apoyado por la Alianza Democrática para la Reforma (UDAR), en unas elecciones que se celebraron sin incidentes, salvo en el Donbass, con una participación total algo superior al $55 \%$.

Una de las primeras medidas del nuevo presidente fue crear pasillos humanitarios en el Este con el fin de permitir a los civiles abandonar las zonas de conflicto $^{23}$. El derribo de un avión de transporte ucraniano unos días más tarde, por rebeldes armados prorrusos en Lugansk, causando la muerte de 49 soldados, no impidió que el nuevo presidente Porochenko anunciara un plan de paz para el este del país, que incluía un diálogo con los rebeldes con el fin de restaurar la integridad de Ucrania. La declaración unilateral de alto el fuego ucraniano, con una duración inicial de una semana, fue ampliada hasta finales de junio, pero no más allá de esta fecha a pesar de la presión francoalemana sobre las autoridades ucranianas, que creyendo que podían ganar militarmente el conflicto en poco tiempo, intensificaron la ofensiva contra los rebeldes, retomando el control del aeropuerto de Lugansk y re-

${ }^{21}$ Según datos de las Comisiones Electorales correspondientes, en Donetsk se emitieron 2.252 .876 votos a favor de la independencia $(89,70 \%$ del total) frente a 256.040 en contra (un $10,12 \%$ del total). En la provincia de Lugansk, con una participación de alrededor del $75 \%$ del censo, algo mayor que en Donetsk, el 96,2\% de los votantes se pronunció a favor de la independencia. Cfr. El País, 13 de mayo de 2014.

${ }^{22}$ De un total de 23 candidatos, Porochenko obtuvo el 55,9\% de los votos por lo que no fue necesaria una segunda vuelta. El segundo fue Yulia Timoshenko del partido «Patria»que obtuvo unos resultados muy discretos $(12,9 \%)$. El 25 de mayo se celebraron igualmente elecciones municipales en veintisiete ciudades, entre las que se encontraban Kiev, donde ganó Vitali Klitschko, uno de los líderes de las revueltas del Maidán, y Odessa. Cfr. Fondation Robert Schuman, Observatoire des Élections en Europe. Disponible en: http://www.robert-schuman. eu/fr/oee/1512-petro-porochenko-triomphe-des-le-1er-tour-1-election-presidentielle-en-ukraine (Fecha de la última consulta: 6-3-2015)

${ }_{23}$ Petro Poroshenko, President of Ukraine. Official website: «The President instructed to establish an escape corridor for civilians willing to move from the territories where the counterterrorist operation is being held». Disponible en: http://www.prezident.gov.ua/en/ news/30504.html (Fecha de la última consulta: 6-3-2015) 
abriendo numerosas acciones militares en varios frentes, que les llevaron a reducir el territorio controlado por los prorrusos a una tercera parte aproximadamente de las provincias de Donetsk y Lugansk, hacia mitad del mes de julio de 2014.

El derribo de un avión de la compañía Malaysia Airlines el día 17 de julio de 2014, mientras sobrevolaba una zona de conflicto en Donetsk causando la muerte de casi trescientas personas de varias nacionalidades, entre los que se encontraban numerosos ciudadanos de la Unión, vino a añadir un grado de mayor dramatismo al conflicto al tratarse de víctimas civiles. La UE, Estados Unidos y numerosos Estados y organizaciones internacionales condenaron firmemente el atentado que sufrió el vuelo MH17, exigiendo a los grupos rebeldes que operaban en la zona, un acceso inmediato y seguro al lugar donde cayeron los restos del avión, así como la puesta en funcionamiento de un corredor de seguridad con el fin de identificar las víctimas y recuperar los restos y efectos personales de las personas fallecidas para poder proceder a su rápida repatriación.

El Consejo de Seguridad de las Naciones Unidas, en su Resolución 2166 de 21 de julio de 2014, así como el Consejo permanente de la OSCE, llevaron a cabo un llamamiento para realizar una investigación internacional exhaustiva, transparente e independiente de conformidad con las normas de la aviación civil internacional y en coordinación con la OACI.

En el aspecto militar, la contraofensiva lanzada por los rebeldes prorrusos a finales de agosto de 2014, tras la polémica entrada del convoy ruso de supuesta ayuda humanitaria al que seguirían otros posteriores, hizo retroceder el avance de las tropas ucranianas de las anteriores semanas, lo que la OTAN y el Gobierno de Kiev atribuyeron a la implicación directa de Rusia en el conflicto, denunciando una incursión de tropas rusas en la provincia de Donetsk. Con esta nueva situación, marcada por las victorias militares de los separatistas prorrusos ${ }^{24}$, se llegó al inicio de las conversaciones de Minsk a principios de septiembre auspiciadas por la OSCE y en la que participaron Rusia, los rebeldes del Este de Ucrania y el Gobierno de Kiev.

24 Además de recuperar el aeropuerto de Lugansk, los rebeldes conquistaron la estratégica colina de Saur-Monguila que controla el paso hacia Donetsk y Lugansk, así como la también estratégica ciudad de Novoasovsk que abría el camino de los rebeldes hacia Mariúpol, la segunda ciudad de Donetsk y su principal puerto en el mar de Azov. Otras localidades situadas más al norte, como Strarobéshevo y Amvrosievka, cayeron igualmente en manos rebeldes. 


\section{De Minsk I (5 de septiembre de 2014) a Minsk II (12 de febrero de 2015)}

Las recientes victorias militares de los rebeldes separatistas prorrusos, marcaron por lo tanto el inicio de las conversaciones de Minsk, que tras varias jornadas de negociaciones $\left({ }^{25}\right)$ llevaron el 5 de septiembre de 2014 a la firma de un protocolo de doce puntos, entre los que destacaba la declaración de tregua inmediata verificada por la OSCE, que controlaría igualmente la frontera entre Ucrania y Rusia así como la creación de una zona de seguridad en las zonas fronterizas; liberación inmediata de todos los presos y personas detenidas ilegalmente; descentralización del poder y creación de un gobierno autónomo en las regiones de Donetsk y Lugansk, en las que, por otra parte, se adoptarían medidas para mejorar la situación humanitaria, y posteriormente se celebrarían elecciones locales anticipadas ${ }^{26}$. El 19 de septiembre fue adoptado un memorandum que clarificaba la aplicación del protocolo ${ }^{27}$ mientras las violaciones esporádicas del alto el fuego seguían produciéndose, aunque en líneas generales se respetaba al no producirse ninguna acción armada de gran alcance.

La adopción de una Ley por parte del Parlamento ucraniano sobre la concesión de un estatus especial a las provincias de Donetsk y Lugansk, controladas parcialmente por los rebeldes, fue calificada positivamente por Rusia, lo que parecía abrir una nueva fase de arreglo del conflicto. La Ley incorporaba un paquete de medidas como la constitución de un gobierno temporal con una duración de tres años, que permitiría la celebración de elecciones en las zonas separatistas para elegir a sus representantes ante el Gobierno central, así como el uso de la lengua rusa y el establecimiento de relaciones económicas con las provincias rusas limítrofes. Además, se contemplaba una amnistía para todos los combatientes separatistas y miembros

${ }^{25}$ En las que participaron el expresidente ucraniano Leonid Kuchma; los líderes de las provincias rebeldes, Zajárchenko de Donetsk y Plotnikski de Lugansk; el embajador de Rusia en Kiev, Mijaíl Zurábov y la suiza Heidi Tagliavini, en representación de la OSCE. Los negociadores partieron de un plan de paz de siete puntos, propuesto por el presidente ruso Vladimir Putin, cuyos principales aspectos serían recogidos en el plan de doce puntos consensuado entre todas las partes.

${ }^{26}$ Entre otras medidas, como la continuación de un diálogo nacional inclusivo, la entrega a la justicia de las personas implicadas en determinados acontecimientos o la retirada de las armas pesadas y de los mercenarios, así como la adopción de un programa de recuperación económica en el Donbass.

27 Como la retirada por ambas partes del armamento pesado, a una línea fijada en quince kilómetros atrás de la zona de contacto, de tal forma que la zona desmilitarizada tuviera una amplitud de treinta kilómetros; prohibición de vuelos de los aviones de combate y de las operaciones militares ofensivas en toda la zona de seguridad; retirada de todos los mercenarios extranjeros de la zona de conflicto y, finalmente, la creación de una misión especial de la OSCE para supervisar la aplicación del Protocolo de Minsk. 
de los gobiernos autoproclamados, que excluía a los implicados en crímenes graves, entre los que incluía el derribo del avión de Malaysia Airlines, y se concedía un plazo de un mes para que los rebeldes depusieran las armas y pudieran integrase en la policía local que se organizaría en ese mismo plazo.

No obstante, y a pesar de que todo parecía indicar que esta Ley podría permitir avanzar en la resolución progresiva del conflicto a través de la aplicación de los acuerdos de Minsk, los rebeldes prorrusos la consideraron insuficiente manifestando que el objetivo de crear un Estado era para ellos irrenunciable. Al margen de esta cuestión, dos factores contribuyeron decisivamente a que el plan de paz fracasara en las siguientes semanas a su adopción:

En primer lugar, aunque no hubo ofensivas de gran envergadura desde los acuerdos de Minsk, prácticamente a diario se registraron combates, principalmente en el aeropuerto de Donetsk, pero también en otras zonas como Debaltseve y la ciudad de Schastia en Lugansk, incrementándose significativamente el número de víctimas desde la tregua hasta alcanzar la cifra de 3.660, según el Alto Comisionado de las Naciones Unidas para los Derechos Humanos ${ }^{28}$.

En segundo lugar, la convocatoria de elecciones legislativas anticipadas en Ucrania para el 26 de octubre de 2014, que serían ampliamente ganadas por los partidos prooccidentales ${ }^{29}$ fue rechazada por los rebeldes del Donbass que además de proclamar que no participarían en las mismas, convocaron sus propias elecciones para el 2 de noviembre siguiente, a las que Rusia declaró rápidamente su disposición a reconocer así como a colaborar

28 Según un informe de la Oficina del Alto Comisionado de las Naciones Unidas para los Derechos Humanos publicado en Ginebra el 9 de octubre de 2014, unas 3.660 personas habrían pedido la vida entre el 15 de abril y el 6 de octubre de dicho año como consecuencia del conflicto, de los cuales 331 corresponderían al período comprendido entre que fue decretado el alto el fuego, el 5 de septiembre, y el 6 de octubre, que apenas supone un mes. Además, el informe contabilizaba un total de 8.756 heridos, así como una elevada cifra de desplazados internos que según fuentes gubernamentales alcanzaría 375.792 personas. El informe indicaba igualmente que la población civil era la principal afectada por el conflicto y que ambas partes habrían perpetrado violaciones de los derechos humanos y del derecho internacional humanitario. A principios del mes de marzo de 2015, la cifra de víctimas del conflicto superaría la cifra de seis mil, según el mismo organismo. Cfr. http://www.ohchr.org/sp/Pages/ WelcomePage.aspx

${ }^{29}$ Con una participación total del 51,2\% (ni los antiguos doce distritos electorales de Crimea, ni hasta dieciséis de las provincias de Donetsk y Lugansk celebraron elecciones, contabilizando un total de más de 4,5 millones de ucranianos que no pudieron votar), la victoria fue para el Frente Popular del primer ministro Yatseniuk $(22,22 \%)$, seguido del «Bloque Petro Poroshenko» $(21,82 \%)$ del presidente. Para un análisis de dichas elecciones, cfr. RUIZ GONZÁLEZ, F., «Ucrania: revolución y guerra civil...», op. cit., nota 3, pp. 27 y s.s. 
con las autoridades surgidas de dicha convocatoria, lo que justificaba como cumplimiento de los acuerdos de Minsk. Sin embargo, en realidad los contradecía, dado que si bien aquéllos establecían la celebración de elecciones en las provincias rebeldes, aquellas debían ser de carácter «local» y celebrarse «de acuerdo con la ley de Ucrania sobre el estatus temporal de autogestión local», también conocida como «ley del estatus especial», que había sido aprobada por el Parlamento ucraniano tan sólo unas semanas antes.

Celebradas las elecciones en los territorios ocupados por los rebeldes y tras la toma de posesión de los nuevos presidentes de las autoproclamadas repúblicas populares de Donetsk (RPD) y Lugansk (RPL), los acuerdos de Minsk se convirtieron en «papel mojado», aunque ciertamente éstos ya se encontraban en crisis dado que no había habido progreso en ninguno de sus puntos, en particular ni en el régimen de alto el fuego y consiguiente retirada de armas pesadas, ni en la creación de la zona desmilitarizada, ni en la liberación de rehenes así como tampoco en el cierre de la frontera estatal entre Rusia y Ucrania.

A partir de este momento la situación fue deteriorándose progresivamente, temiéndose por la vuelta a la guerra total, tal como se indicó en la reunión de urgencia del Consejo de Seguridad el 12 de noviembre de 2014 convocado a petición de Estados Unidos para examinar las denuncias de incursiones militares rusas en el Este de Ucrania, confirmadas por la OTAN y la OSCE, que acababa de desplegar una misión de 261 observadores en dicha zona.

La escalada bélica provocada por las nuevas ofensivas lanzadas por el Gobierno de Kiev contra las zonas rebeldes, en particular Donetsk, y contestadas por los separatistas prorrusos ampliando sus acciones a otras zonas como Mariúpol, determinaron un escenario de violencia desconocido desde el verano anterior que se cebó de nuevo en la población civil, a pesar de los fracasados intentos de celebrar una nueva ronda de conversaciones por parte del denominado grupo de contacto, formado por Ucrania, Rusia y los representantes de los rebeldes, bajo los auspicios de la OSCE.

Con el objetivo de evitar una guerra abierta en Ucrania con consecuencias quizás irreversibles para toda Europa, la canciller alemana y el presidente francés elaboraron un plan de paz basado en los anteriores acuerdos de Minsk, que presentaron por separado al presidente de Ucrania y de $\mathrm{Ru}-$ sia, en un intento de frenar la escalada bélica que supondría el envío de armas al Ejército de Ucrania que ya sopesaba Estados Unidos. La intensificación de los combates precedió a la cumbre de Minsk, que finalmente pudo

30 Centro de Noticias ONU, «ONU considera responsabilidad colectiva garantizar estabilidad en Ucrania». Disponible en: http://www.un.org/spanish/News/story. asp?NewsID=30968\#.VQ_P_vyG9_c (Fecha de la última consulta: 9-3-2015). 
ser convocada por el llamado «cuarteto de Normandía» (Rusia, Ucrania, Francia y Alemania), para el día 12 de febrero de 2015.

El nuevo acuerdo alcanzado tras diecisiete horas de conversaciones entre los dirigentes de los cuatro países ${ }^{31}$, que posteriormente sería firmado también por los líderes de las dos regiones separatistas y la OSCE, estableció un alto el fuego que entraría en vigor el día 15 de febrero y que además incluía una serie de medidas, entre las que destacan, entre otras, la retirada del armamento pesado y la creación de una amplia zona de seguridad de al menos cincuenta kilómetros de amplitud; retirada de las tropas extranjeras así como de las fuerzas irregulares presentes en la zona de conflicto; establecimiento de corredores humanitarios; intercambio de prisioneros de guerra y concesión de una amnistía para los que hubieran combatido en las regiones rebeldes; restablecimiento de las relaciones económicas entre Kiev y las regiones rebeldes, incluyendo el pago de pensiones y otras ayudas sociales por parte del Gobierno ucraniano a los habitantes del Donbass; reforma constitucional para proporcionar una descentralización que establezca un estatuto especial para las regiones del este antes de finales de 2015 y celebración de elecciones de carácter local en dichas regiones consensuadas entre el Gobierno y los representantes de las regiones rebeldes y, finalmente, y condicionado al cumplimiento de las condiciones del acuerdo, restablecimiento del control ucraniano sobre la frontera con Rusia a finales del presente año.

Como puede observarse, el contenido de tales acuerdos no difiere prácticamente de los firmados en septiembre en la misma ciudad de Minsk. Como entonces, Minsk II obedece a un intento desesperado, ahora por la iniciativa francoalemana, de evitar una escalada bélica que arrastre a una intensificación irreversible del conflicto, frente a unos rebeldes sostenidos por Rusia y que fortalecidos por sus recientes victorias militares se hallan poco dispuestos a ceder ante el Gobierno central en sus pretensiones separatistas así como de una eventual unión a Rusia. El acuerdo, a todas luces necesario, nació por lo tanto frágil, como se demostró en los días siguientes a su conclusión.

En efecto y a pesar de que en líneas generales pareció respetarse en su inicio, tan sólo una semana más tarde de haberse firmado, los rebeldes infligieron una severa y humillante derrota al ejército de Kiev al tomar la estratégica ciudad de Debáltsevo que posibilita la interconexión ferroviaria entre

31 OSCE, Package of Measures for the Implementation of the Minsk Agreements. Disponible en http://www.osce.org/cio/140156. (Fecha de la última consulta: 9-3-2015). Sobre tales acuerdos puede verse igualmente, CORRAL HERNÁNDEZ, D., «La OSCE en Ucrania, una garantía de futuro», Instituto Español de Estudios Estratégicos. Documento Opinión, $n{ }^{o} 20 / 15,17$ de febrero de 2015 . 
los dos bastiones rebeldes de Donetsk y Lugansk. Esta acción fue calificada por Ucrania, la UE y Estados Unidos como una clara violación del alto el fuego, lo que demostró la fragilidad del acuerdo, que pese a todo empezó a aplicarse en lo relativo a la retirada, lenta pero progresiva, de las armas pesadas o al intercambio de prisioneros, abriendo una nueva fase de una cierta esperanza, amenazada, sin embargo, por las continuas escaramuzas que todavía existen en el frente de batalla, y de las que se acusan recíprocamente ambas partes.

\section{Impacto del conflicto de Ucrania en las relaciones de Rusia con la UE y con la OTAN}

\section{Impacto en las relaciones de la Federación Rusa con la Unión Europea}

El apoyo ruso a los rebeldes del Donbass, ha obligado a la Unión a redefinir sus relaciones con Rusia, adoptando una posición que intenta combinar una actitud de firmeza, a través de las sanciones, con un intento de no romper todos los canales de comunicación con su poderoso vecino del Este.

Las sanciones impuestas por la UE y Estados Unidos contra Rusia están afectando su economía en varios frentes. En primer lugar, las sanciones que se dirigen al sector financiero y a la industria militar afectarán a los programas de modernización que el Gobierno ruso había diseñado en ambos sectores para los próximos años. En segundo lugar, el sector energético acusará igualmente las medidas restrictivas.

Por lo que respecta al sector financiero, verdadero talón de Aquiles de la economía rusa por su dependencia exterior y uno de los objetivos más definidos de las sanciones occidentales, conviene señalar que si a la caída de los precios de los hidrocarburos añadimos el importante vencimiento de la deuda exterior en los próximos meses, así como el saldo negativo de los flujos de capital extranjero, es previsible que las sanciones tengan un efecto considerable sobre la economía rusa dada la interdependencia de ésta y de su sector financiero con la economía global.

A pesar de que el país cuenta con reservas suficientes para hacer frente a las dificultades de acceso a la financiación y de que puede recurrir a mecanismos como imprimir más moneda con los riesgos asociados de depreciación y de incremento de la inflación, las recientes subidas de los tipos de interés por parte del Banco central ruso para frenar la caída del rublo, están teniendo repercusiones en el encarecimiento de la financiación para empresas y bancos con las consiguientes impactos negativos en la inversión y las importaciones. Las dificultades para el acceso al crédito y a la financiación 
provocarán que grandes proyectos de modernización en distintos sectores de la economía rusa, se verán postergados, en particular en el sector industrial, que al no ser competitivo necesita la inversión extranjera para revertir la situación. Además de frenar la inversión, las sanciones impedirán la entrada de material y tecnología clave para aumentar la competitividad del sector industrial.

Sin embargo, y dado que las sanciones son de «ida y vuelta» no sólo por los efectos de las sanciones occidentales en la propia economía europea sino también por las contramedidas adoptadas por Rusia en agosto de 2014, que afectan a la importación de determinados productos agrarios y agropecuarios procedentes de la UE y de otros países occidentales, conviene igualmente tener presentes los efectos que aquellas están provocando en la propia Unión, aunque la capacidad de ésta de adaptarse a unas mayores restricciones comerciales es superior que la que presenta Rusia, al margen del sector energético, en el que se detecta recientemente un mayor esfuerzo de la UE y de sus Estados miembros por reducir la dependencia de su vecino del Este.

Aunque, como demuestra la historia, es discutible que este tipo de sanciones consigan el objetivo político y sean verdaderamente efectivas ${ }^{32}$, en este caso doblegar la voluntad rusa de intervenir en Ucrania, el hecho de que aquellas tengan una doble dirección tras las contramedidas rusas, genera una serie de implicaciones de contenido variable ${ }^{33}$.

En primer lugar, que a pesar de que las sanciones también han afectado a las empresas europeas, aquéllas están teniendo un impacto mayor en la economía rusa. En segundo lugar, que las sanciones, recientemente prorrogadas por la Unión tras la escalada bélica que precedió a los acuerdos de Minsk II, pueden ser intensificadas por la UE si la situación se deteriorase todavía más, pudiendo alcanzar a la exclusión de Rusia del sistema de comunicaciones financieras SWIFT o a las inversiones en el sector gasístico ${ }^{34}$.

32 Vid. en este sentido ERIKSSON, M., West's Sanctions against Russia: Grand Strategy in the Making?, RUFS Briefing, FOI, Swedish Defence, Research Agency, May 2014.

33 Como afirma la profesora Araceli Mangas, además de que en este caso las medidas de retorsión dañan a ambas partes y en particular la recuperación económica de la UE, en general sólo son eficaces cuando los Estados que las adoptan son mucho más fuertes que sus destinatarios. Por otra parte, las sanciones occidentales han lanzado a Rusia en brazos de su vecino chino, reforzando el eje asiático, que es justamente lo que menos conviene a los intereses europeos. MANGAS MARTÍN, A., «Restaurar y redefinir las relaciones con Rusia», Real Instituto Elcano, $A R I$ n. ${ }^{\circ} 55 / 2014$, de 17/11/2014, p. 5.

${ }^{34}$ Cfr. NIBLETT, R., «Quelle politique de 1'Europe devrait-elle adopter face à la Russie?». Fondation Robert Schuman. Policy Paper. Question d'Europe, n. ${ }^{\circ} 349,23$ de marzo 2015. 


\section{Impacto en las relaciones de la Federación Rusa con la OTAN}

Sin lugar a dudas, el conflicto de Ucrania ha cambiado absolutamente los parámetros en que se han basado las relaciones bilaterales durante los últimos años ${ }^{35} \mathrm{y}$ ha generado desconfianzas mutuas, que han determinado, por un lado, que la OTAN reoriente su foco de atención hacia Europa, en particular a su zona más oriental tras varios años centrada en Afganistán, y por el lado ruso, se ha generado un resentimiento hacia Occidente que ha condicionado totalmente sus acciones en los últimos meses.

El intento de recomponer las relaciones tras el conflicto de Georgia, entró en una nueva fase en marzo de 2014, tras la anexión de Crimea y el inicio de las operaciones militares en el Donbass, cuyo apoyo por parte de Moscú, supuso la suspensión de la cooperación, aunque se decidió mantener los canales básicos de comunicación. La presencia de más de 30.000 soldados rusos cerca de las fronteras con Ucrania tras la anexión de Crimea, hizo saltar las alarmas en los cuarteles generales de la OTAN donde planeó de nuevo el recuerdo de los tiempos de la disuasión y de la guerra fría, dos décadas después de la disolución del Pacto de Varsovia.

Tras el anuncio de abandonar Afganistán, la OTAN debe afrontar nuevos desafíos derivados de las consecuencias de los recortes en defensa de sus socios, lo que preocupa en particular a Estados Unidos, pero también derivados de los nuevos intereses norteamericanos en el Pacífico sin dejar de afianzar la credibilidad de los nuevos socios del Este ante la amenaza rusa que éstos perciben de forma directa. Ello ha conllevado un reenfoque hacia la seguridad colectiva en Europa lo que implica aumentar la presencia en el mar Báltico, en el mar Negro así como el envío de aviones a Polonia, Rumania y los Estados Bálticos en lo que ha constituido una revisión y ampliación de los planes de contingencia de la organización.

La OTAN ha alertado, durante el desarrollo del conflicto, del avance ruso en el Donbass, confirmando el despliegue de más de mil soldados rusos en dicha región tras haber cruzado ilegalmente la frontera a mediados de agosto de 2014 en lo que interpretó como un intento de Rusia de evitar la derrota de los rebeldes tras haber perdido éstos bastantes posiciones en

35 Tanto la OTAN como posteriormente la UE han intentado identificar espacios de interés común con Rusia en los que intensificar la cooperación iniciada ya en la década de los noventa, a pesar de los inevitables desencuentros y divergencia de intereses, como quedó patente en el conflicto de Georgia, y ahora en Ucrania. Para un análisis de las relaciones de la OTAN con Rusia, vid. BLANC ALTEMIR, A., «Rusia y la arquitectura europea de seguridad», en RAMÓN CHORNET, C. (coord.), La acción colectiva del uso de la fuerza. Nuevos escenarios, «nuevos principios» de actuación en el orden internacional. Tirant lo Blanch, Valencia, 2012, pp. 375-425. 
las últimas semanas. Para la OTAN, la exitosa contraofensiva de los rebeldes antes de los primeros acuerdos de Minsk a principios de septiembre obedeció a este apoyo directo ruso. A pesar de que Rusia ha negado sistemáticamente cualquier participación directa en el conflicto, lo que parece evidente y nadie discute es la participación de nacionalistas rusos en los destacamentos rebeldes, que el propio primer ministro de la autoproclamada república de Donetsk ha llegado a cuantificar en alguna ocasión entre tres y cuatro mil ${ }^{36}$.

La actitud rusa en el conflicto del Donbass ha provocado un cambio en la percepción del entorno de seguridad de la OTAN que ahora percibe a Rusia como una amenaza potencial para la seguridad de sus miembros, como lo demuestran en particular dos hechos:

En primer lugar, el mismo día en que se estaban firmando los primeros acuerdos de Minsk (5 de septiembre de 2014), los jefes de Estado y de Gobierno de los países aliados decidían en la reunión de Newport (Gales, Reino Unido) la creación de una fuerza de acción inmediata con capacidad de acción contra cualquier agresor potencial, pero con el objetivo específico de disuadir a Rusia. La fuerza de intervención rápida, con una dotación de alrededor de cuatro mil efectivos, tendrá su cuartel central en Polonia, aunque dispondrá igualmente de otros de apoyo en diferentes países del Este. Para favorecer el despliegue inmediato de tales efectivos, la fuerza de intervención debe contar con equipos instalados en el este de Europa. Sin embargo y con el fin de no violar el Acta Fundacional sobre las Relaciones, Cooperación y Seguridad Mutuas entre la OTAN y la Federación Rusa, de 27 de mayo de 1997 que prohibía la instalación de bases militares permanentes en la región oriental, se prevé la rotación sucesiva de las unidades que de esta forma estarían listas en poco tiempo sin la necesidad de constituirse en unidades fijas y permanentes, evitando de esta forma violar dicho documento.

En segundo lugar, y directamente relacionado con la creación de esta fuerza de intervención rápida, debe señalarse el esfuerzo que la OTAN está realizando en el Este de Europa. En efecto y tras la anexión de Crimea los países de la Alianza, que desde el ingreso de los países bálticos en 2004 asumieron de forma rotatoria la vigilancia de su espacio aéreo, han cuadriplicado el número de aviones desplegados en el mar Báltico, hasta un total de dieciséis (cuatro en Estonia, cuatro en Letonia y ocho en Lituania), países que, por otra parte han incrementado notablemente sus gastos de defensa al percibir una mayor amenaza a su seguridad, tras el conflicto de Ucrania.

36 En unas declaraciones realizadas al canal oficialista Rossiya 24. Cfr. El País, 28 de agosto de 2014. 
La reacción de Rusia no se hizo esperar pues tanto el discurso del Estado de la Nación, pronunciado por el presidente Putin el pasado 4 de diciembre, como la nueva doctrina militar adoptada el 25 del mismo mes, han estado condicionados por la grave crisis de Ucrania y el enfrentamiento con Occidente $^{37}$, considerando el avance de la OTAN y el despliegue del sistema antimisiles en Europa, como los principales peligros para la seguridad nacional rusa. La consecuencia de todo ello es el refuerzo del despliegue militar ruso tanto en Crimea como en el Báltico - donde se han incrementado sensiblemente el número de incidentes aéreos, algunos de los cuales producidos por violaciones del espacio aéreo europeo- además del Ártico, con un incremento del gasto en defensa, que para 2016 se calcula superará la suma combinada de Francia y Alemania ${ }^{38}$.

\section{Conclusiones}

Además del inestable flanco Sur, las amenazas al entorno estratégico de la Unión Europea provienen de su flanco Este, desde la progresiva implicación de Rusia en el conflicto de Ucrania, primero en Crimea y después en el Donbass. Ello constituye una novedad pues a pesar de que las relaciones entre Rusia y la UE siempre se han caracterizado por una enorme complejidad, ambos actores han ido construyendo una tupida red de relaciones poliédricas, partiendo de una visión pragmática desde la recíproca constatación de la interdependencia y complementariedad en torno a unos intereses frecuentemente compartidos aunque fuera en contextos y ámbitos determinados.

Es evidente que el conflicto de Ucrania ha trastocado profundamente las relaciones bilaterales pacientemente construidas a lo largo de los últimos años. Más allá de las sanciones y sus efectos, interesa a ambas partes el diseño a largo plazo, pues una vez que el conflicto se estabilice y se asimile definitivamente el no retorno de Crimea a la soberanía ucraniana, es previsible que las aguas vuelvan a su cauce. No obstante, es evidente que

37 Sin duda, se percibe un cambio sustancial en el tono del discurso de Vladimir Putin sobre el estado de la Nación de 2014 respecto del pronunciado en 2013, pues si bien en el del año anterior el presidente Putin incidió en las iniciativas coordinadas con Occidente, aunque sin eludir las diferencias, en el relativo a 2014 se ha centrado totalmente en el enfrentamiento con Occidente, lo que reflejaría el evidente resentimiento que se ha instalado en los círculos de poder rusos ante la OTAN, Estados Unidos y la UE. Cfr. RUIZ GONZÁLEZ, F.J., «Rusia y el mundo según Putin: el discurso del estado de la Nación». Instituto Español de Estudios Estratégicos. Documento Opinión, n. ${ }^{\circ}$ 144/2014, de 15 de diciembre de 2014.

38 Cfr. IHS Jane's International Defence Review. Disponible en http://www.janes.com/ magazines/ihs-janes-international-defence-review (Fecha de la última consulta: 12-3-2015). 
el conflicto de Ucrania tardará en cicatrizar pues ha trastocado el orden establecido en Europa tras la implosión de la URSS en 1991 y ha generado profundas desconfianzas en la UE pero también recelos en la parte rusa. El clima de cooperación vigente en el pasado ha dado paso a un escenario de competición que se manifiesta en Europa Oriental y en Ucrania en particular, donde los antiguos socios se contemplan como competidores por lograr una mayor esfera de influencia.

Ante el posible escenario de que la situación en Ucrania se congele, e incluso de que empeore, la Unión debe reservarse un cierto margen de maniobra, sin implicarse más a fondo proporcionando armas a Kiev, como algunas voces reclaman, en particular Estados Unidos y ciertos gobiernos europeos. En sus relaciones bilaterales con su gran vecino oriental, la UE no puede ignorar que Rusia sigue siendo una potencia militar y energética de primer orden así como un actor muy relevante en la esfera internacional, cuya participación como miembro permanente del Consejo de Seguridad le permite estar presente en los temas más candentes de la sociedad internacional en el momento presente. Precisamente por ello y con el fin de no romper los puentes de comunicación con Rusia, es conveniente que la UE, sin dejar de mantener la firmeza, explore los posibles canales de cooperación con su poderoso vecino del Este, en particular en temas de interés común como la lucha contra el terrorismo yihadista que representa el Estado Islámico y Al Qaeda, el futuro de Afganistán o la cuestión del programa nuclear iraní.

Sin lugar a dudas la estabilidad de la región y de todo el continente europeo depende muy directamente de la capacidad de ambas partes, Rusia y Unión Europea, de resolver los problemas derivados de la situación creada por el conflicto de Ucrania. Precisamente por ello la UE, a pesar de que existen otros actores en presencia como Estados Unidos, debe actuar estratégicamente pues está en juego no sólo la forma en que el desarrollo del conflicto afectará en el futuro la estabilidad europea, sino también sus relaciones con Rusia y con toda la vecindad oriental. 
